\title{
Effects of dantrolene on calcium-induced calcium release from the sarcoplasmic reticulum in human skeletal skinned fibers
}

Keiko Mukaida, Sachiko Otsuki, Takashi Kondo, Toshimichi Yasuda, Noboru Saeki, Masashi Kawamoto

Anesthesia, Hiroshima Prefecture Rehabilitation Center, Higashi-Hiroshima, Japan.

Department of Anesthesiology and Intensive care, Hiroshima University Hospital, Hiroshima, Japan

\section{Background}

Dantrolene (Dan) is the only drug available to treat malignant hyperthermia $(\mathrm{MH})$ crisis, a pharmacogenetic disorder caused by increased calcium $(\mathrm{Ca})$ release from the sarcoplasmic reticulum $(\mathrm{SR})$ via ryanodine receptor 1 (RYR1). The Ca-induced Ca release (CICR) rates from the SR were found to be accelerated in the skeletal muscles of patients with $\mathrm{MH}^{1}$ ). The effects of Dan on CICR in skinned fibers are controversia|2)3), and have not been studied in the skeletal muscles of patients with $\mathrm{MH}$.

\section{Goal of Study}

We investigated the effects of Dan on the CICR rate in the skeletal muscles of patients predisposed to $\mathrm{MH}$. Materials and Methods

This study was approved of the local ethics committees (Hiroshima University and Hiroshima Prefecture Rehabilitation Center )

Muscle samples were obtained, with prior written consent, from 11 individuals to determine their predisposition to $\mathrm{MH}$.

The CICR rates were measured in chemically skinned fibers achieved by Endo's method. Calcium ions $\left[\mathrm{Ca}^{2+}\right]$ at five different concentrations $(0,0.3,1.0,3.0$, and 10 $\mu \mathrm{M})$, were used to induce $\mathrm{Ca}^{2+}$ stimulation via RYR1. The effects of Dan-treatment $(50 \mu \mathrm{M})$ on CICR rates were assessed at five different $\mathrm{Ca}^{2+}$ concentrations, under magnesium-free conditions, at $20^{\circ} \mathrm{C}$. Dan $(50 \mu \mathrm{M})$ was added in 4-2 and 5 of CICR procedure (Table 1 ).

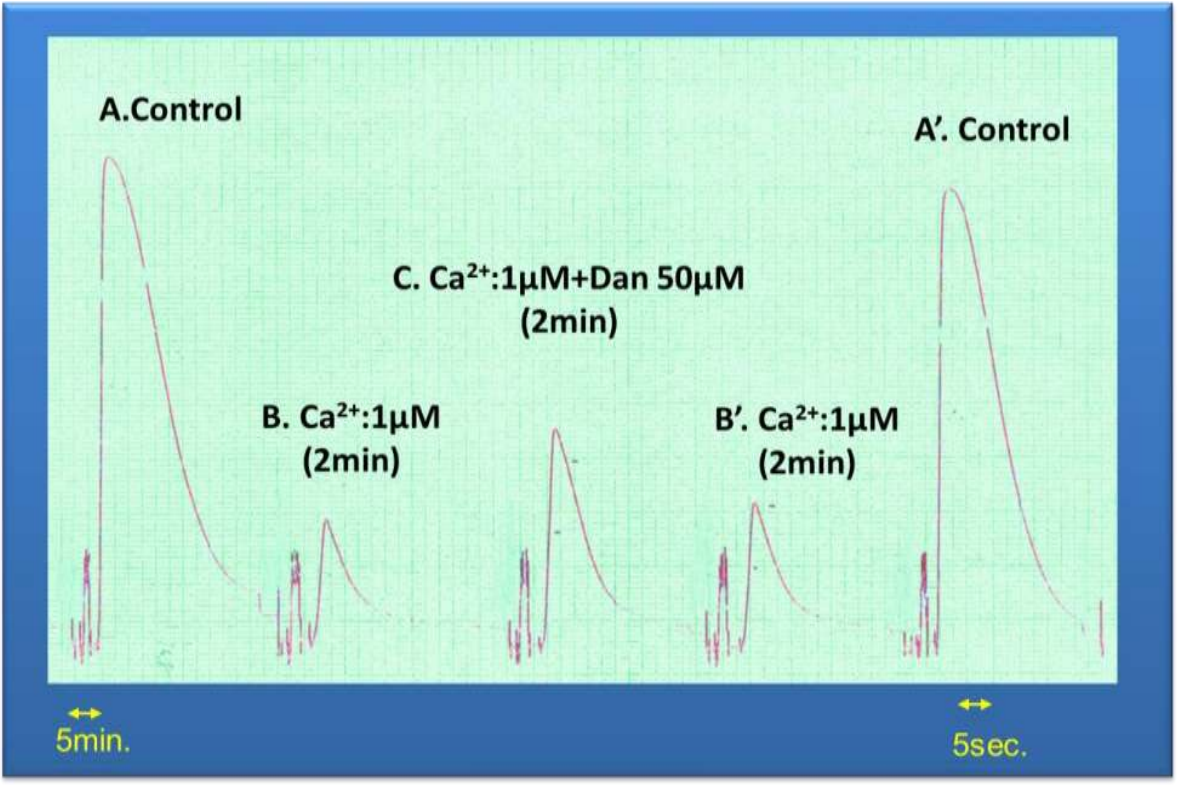

Fig. 1 A representative trace of time-tension curve
Table 1. Outline of CICR procedure and constitution of the solutions

\begin{tabular}{|c|c|c|c|c|c|c|c|}
\hline CICR procedure & solutions & $\begin{array}{l}\mathrm{Mg}^{2+} \\
\mathrm{mM}\end{array}$ & $\begin{array}{l}\text { MgATP } \\
\text { mM }\end{array}$ & $\begin{array}{l}\mathrm{Ca}^{2+} \\
\mu \mathrm{M}\end{array}$ & $\begin{array}{l}\text { EGTA } \\
\text { mM }\end{array}$ & $\begin{array}{l}\text { PIPESm } \\
\text { M }\end{array}$ & $\begin{array}{l}\text { Others } \\
\mathrm{mM}\end{array}$ \\
\hline 1. Relaxing & Relaxing solution & 1.5 & 3.5 & 0 & 2 & 20 & \\
\hline 2. Loading $\mathrm{Ca}^{2+}$ into SR with ATP & Ca loading solution & 1.5 & 3.5 & 0.2 & 10 & 20 & \\
\hline 3. Sop loading $\mathrm{Ca}^{2+}$ with high EGTA & $\begin{array}{l}\text { relaxing solution high } \\
\text { EGTA }\end{array}$ & 1.5 & 3.5 & 0 & 10 & 20 & \\
\hline 4-1. Washout ATP & ATP free solution & 1.5 & 0 & 0 & 2 & 20 & \\
\hline 4-2. Washout ATP and $\mathrm{Mg}^{2+}$ & Mg and ATP free solution & 0 & 0 & 0 & 2 & 20 & \\
\hline $\begin{array}{l}\text { 5. Releasing } \mathrm{Ca}^{2+} \text { from } \mathrm{SR} \text { with } \\
\mathrm{X}\left(\mathrm{Ca}^{2+}\right) \mu \mathrm{M} \text { for } \mathrm{t}^{* *} \mathrm{~min}\end{array}$ & releasing solution & 0 & 0 & $\mathrm{X}^{*}$ & 10 & 20 & \\
\hline 6. stop releasing $\mathrm{Ca}^{2+}$ with Procaine & stop solution & 10 & 0 & 0 & 10 & 20 & Procaine 10 \\
\hline 7. Re-introduce ATP & pre-assay solution & 1.5 & 3.5 & 0 & 0.1 & 20 & Procaine 5 \\
\hline $\begin{array}{l}\text { 8. Releasing all residual } \mathrm{Ca}^{2+} \text { from } \\
\mathrm{SR} \text { with } 25 \mathrm{mM} \text { caffeine }\end{array}$ & assay solution & 0.1 & 1.0 & 0 & 0.1 & 20 & Caffeine 25 \\
\hline
\end{tabular}

EGTA ; ethylene glycol-bis( $\beta$-amino ether) - N, N, N', N'-tetra acetic acid PIPES ; piperazine $-\mathrm{N}, \mathrm{N}^{\prime}$-bis(2-ethanesulfonic acid) $X^{*}=$ free, $0.3,1.0,3.0,10$

$\mathrm{t}^{\star *} \min$; time of releasing $\mathrm{Ca}^{2+}$ in $\mathrm{CICR}$ procedure 5

B : $1 \rightarrow 2 \rightarrow 3 \rightarrow 4-1 \rightarrow 4-2 \rightarrow 5 \rightarrow 6 \rightarrow 7 \rightarrow 8$

C : $1 \rightarrow 2 \rightarrow 3 \rightarrow 4-1 \rightarrow 4-2 \rightarrow 5 \rightarrow 6 \rightarrow 7 \rightarrow 8$

The area under time-tension curve is equal to $\mathrm{Ca}^{2+}$ amount releasing in CICR procedure 8 $S_{A}$ : area of curve $A, S_{B}$ : area of curve $B$. CICR rate $\left(\min ^{-1}\right)=-\left\{\ln \left(S_{A}-S_{B} / S_{A}\right)\right\} / t$
Statistical analyses : The values are expressed as mean $\pm \mathrm{SD}$. A paired t-test and a regression analysis were performed for statistical analysis. Significance was determined at $p<0.05$.

\section{Results}

Table 2. Demographic data

Ten of the 11 individuals were diagnosed with predisposition to $\mathrm{MH}$, based on the accelerated CICR rates (Table 2). Their CICR rates were increased by 2 to 8 -fold compared to the normal rate. Dan-treatment significantly decreased the CICR rates at $\mathrm{Ca}^{2+}$ concentrations of $0.3,1.0,3.0$ and $10 \mu \mathrm{M}$ (Table 3). CICR rates, both pre- and post- Dan-treatment, exhibited a significant correlation (Fig 2).

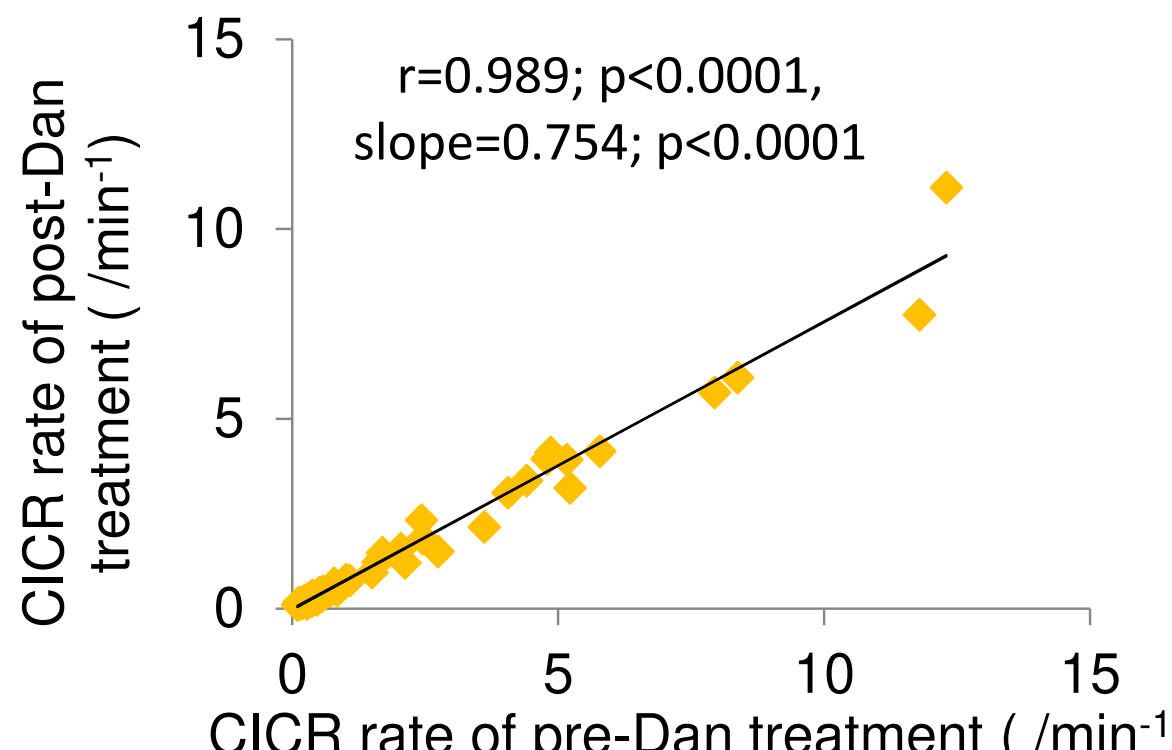

CICR rate of pre-Dan treatment $\left(/ \mathrm{min}^{-1}\right)$

Fig. 2 Correlation of CICR rates,

both pre- and post- Dan-treatment

\begin{tabular}{|c|c|c|c|c|c|c|}
\hline No. & Gender & $\begin{array}{l}\text { Age * } \\
(y)\end{array}$ & Indication for CICR test & $\begin{array}{c}\text { Result of CICR } \\
\text { test }\end{array}$ & $\begin{array}{l}\mathrm{CK}^{*} \\
(\mathrm{IU} / \mathrm{I})\end{array}$ & $\begin{array}{c}\text { Variants detected in } \\
\text { RYR1 }\end{array}$ \\
\hline 1 & male & 33 & $\mathrm{MH}$ reaction (no data) & accelerated & 391 & p. Ala1449val \\
\hline 2 & male & 34 & $\mathrm{MH}$ reaction (no data) & accelerated & 267 & p. Gly341Arg \\
\hline 3 & female & 56 & $\mathrm{MH}$ reaction ( CGS 30 ) & accelerated & 204 & p. Arg614Leu \\
\hline 4 & male & 12 & Suspicion of muscle disease & accelerated & 189 & p.Thr84Met \\
\hline 5 & male & 52 & $\mathrm{MH}$ reaction (no data) & accelerated & 50 & p. Ala5025Gly \\
\hline 6 & female & 73 & Brother died in $\mathrm{MH}$ reaction & accelerated & 297 & p. Gln155Lys \\
\hline 7 & female & 65 & Brother developed an $\mathrm{MH}$ & accelerated & 98 & No variant \\
\hline 8 & male & 40 & Brother died in $\mathrm{MH}$ reaction & accelerated & 276 & No variant \\
\hline 9 & male & 37 & $\mathrm{MH}$ reaction ( CGS 30 ) & accelerated & 322 & No variant \\
\hline 10 & male & 42 & $\mathrm{MH}$ reaction ( CGS 48 ) & accelerated & 305 & p. Ser2345Thr \\
\hline 11 & male & 44 & Idiopathic high CK & not accelerated & 1135 & Not done \\
\hline
\end{tabular}

Table 3. Relative $\mathrm{Ca}^{2+}$ release rates

\begin{tabular}{|cccccc|}
\hline $\mathrm{Ca}^{2+}(\mu \mathrm{M})$ & free & 0.3 & 1.0 & 3.0 & 10.0 \\
\hline Relative $\mathrm{Ca}^{2+}$ release rate & $0.95 \pm 0.18$ & $0.79 \pm 0.22$ & $0.67 \pm 0.087$ & $0.74 \pm 0.11$ & $0.73 \pm 0.13$ \\
\hline P value & 0.0832 & 0.0087 & $<0.0001$ & 0.0001 & 0.0006 \\
\hline
\end{tabular}

Discussion : It was therefore assumed that applying $50 \mu \mathrm{M}$ Dan would decrease the CICR rates by $0.754-$ fold. The inhibitory effect of Dan on CICR may contribute to the suppression of myoplasmic elevation of $\mathrm{Ca}^{2+}$ in $\mathrm{MH}_{\mathrm{H}}$ crisis. 